\title{
Article \\ A Dual Incentive Value-Based Paradigm for Improving the Business Market Profitability in Blockchain Token Economy
}

\author{
Chaopeng Guo $\mathbb{1}$, Pengyi Zhang, Bangyao Lin and Jie Song * \\ Software College, Northeastern University, Shenyang 110000, China; guochaopeng@swc.neu.edu.cn (C.G.); \\ 20185152@stu.neu.edu.cn (P.Z.); 2071292@stu.neu.edu.cn (B.L.) \\ * Correspondence: songjie@mail.neu.edu.cn
}

check for

updates

Citation: Guo, C.; Zhang, P.; Lin, B.;

Song, J. A Dual Incentive

Value-Based Paradigm for Improving the Business Market Profitability in

Blockchain Token Economy.

Mathematics 2022, 10, 439. https://

doi.org/10.3390/math10030439

Academic Editors: José Luis

Miralles-Quirós and Maria Del Mar

Miralles-Quirós

Received: 26 December 2021

Accepted: 28 January 2022

Published: 29 January 2022

Publisher's Note: MDPI stays neutral with regard to jurisdictional claims in published maps and institutional affiliations.

Copyright: (C) 2022 by the authors. Licensee MDPI, Basel, Switzerland. This article is an open access article distributed under the terms and conditions of the Creative Commons Attribution (CC BY) license (https:// creativecommons.org/licenses/by/ $4.0 /)$.

\begin{abstract}
Blockchain solves the problem of mutual trust and consensus in the business market of the token economy. In the existing paradigm of blockchain token economy, there are disadvantages of lacking the incentive mechanism, business applications and virtual token value. These shortcomings reduce consumers' willingness to consume and the profits of the merchants. In this paper, we propose a novel "Dual incentive value-based" paradigm to improve the business market profitability in blockchain token economy. To evaluate our proposed paradigm, we propose a business study case for improving merchants' environment state. In this case, we set up two economic models and make simulations to validate the profitability. The result shows that merchants with the novel paradigm have $32 \%$ more profit compared with those without the paradigm and at most $10 \%$ more profitable than those in existing paradigms.
\end{abstract}

Keywords: blockchain; token economy; incentive mechanism; effects in business market; economic model

\section{Introduction}

A token economy is a complex system of reinforcement, and it offers some medium of exchange (i.e., a token) for the participants to purchase various goods, services, or privileges [1]. The token economy could make the business market more active and profitable than before [2]. Traditional token economy has not fully reflected the characteristics of co-governance and mutual trust [3]. With the development of blockchain technology, many studies believe that the blockchain token economy is a crucial grasp to achieve this goal based on the consensus mechanism and autonomy of the blockchain [4].

Blockchain can be referred as a distributed ledger. Its data and transactions are not under the control of any third party [5]. Each node in the blockchain is equal. The blockchain is considered as persistence, anonymity and auditability by the academia and the industry [6]. In 2008, Satoshi Nakamoto proposed the definition of Bitcoin, where the concept blockchain was firstly proposed. After that, the blockchain experienced three development stages. Blockchain 1.0 resolves Double-spend with distributed transfer in the cryptocurrency transactions such as the Bitcoin. Blockchain 2.0 realized the programmable finance in the financial fields such as the Ethereum. Blockchain 3.0 focuses on using blockchain for various applications in industries other than finance [7]. Currently, Blockchain 3.0 can be well applied in many fields, such as healthcare [8], education [9] and the token economy introduced in this paper. In the blockchain, the token (i.e., Bitcoin) is a medium to stimulate nodes to work for the community more. The incentive mechanism is the approach to offer tokens. The incentive mechanism is a common approach to stimulate roles to work in different scenarios. It is widely used in different fields, such as network communications [10] and IoT [11]. The design of the incentive mechanism is the critical challenge of decentralized token design [12]. Moreover, it increases the blockchain nodes' interests in the token [13]. In the business market, the blockchain token economy can 
enforce a mutually beneficial business environment [14]. In this environment, the values of the token and the incentive mechanism are two essential factors [15].

Existing studies on the blockchain token economy mainly focus on three aspects: new incentive mechanism design, new economic scenario design and new business schema design

- $\quad$ For the first aspect, researchers concentrate on designing new incentive mechanism on the token to stimulate nodes to join in the activities of the community. Barreiro-gomez et al. [16] designed a game theory-based incentive mechanism on the token to research the risks in the token's circulation. Kim et al. [12] introduced the details in a Swiss blockchain's token, including the design of the incentive mechanism. Drasch et al. [17] modeled the associated incentives in a multi-sided blockchain-enabled platform for token value development.

- For the second aspect, researchers import particular economic scenarios in the blockchain token economy. The definitions of novel economies and management methods are necessary. Narayan et al. [18] proposed a tokenizing coopetition in the circular token economy to show how competition could be operationalized and optimized using tokens in a blockchain. Kim et al. [19] proposed a process for building a desirable model of a sustainable growth token economy.

- For the last aspect, researchers introduces the application of tokens in the actual business schema, such as the joint application of Initial Coin Offering (ICO) [20] and tokens. Tonnissen et al. [21] introduced the cluster-analysis of 195 ICOs and identified three different archetypes on ICO tokens.

At present, there is a lack of systematic research on the token economy paradigm. Therefore, we divide the current researches into three categories according to their research directions: the incentive-based paradigm, the new scenario-based paradigm, and the business schema-based paradigm.

However, the three paradigms all have disadvantages. First, the incentive-based paradigm's token cannot be used as a real currency [12,16]. Meanwhile, the current incentive-based paradigm focuses on the single incentive mechanism, leading to limited influence in their scenario. Second, the new scenario-based paradigm lacks applications in the business scenario. It also lacks regulation and control effects to the business market economy. Finally, the business schema-based paradigm lacks the incentive mechanism. It takes terrible effects, such as the virtual tokens scam.

Token economy strengthens the market profit and helps to build mutual trust and cogovernment [22]. The incentive mechanism and the liquidity of tokens are most essential parts. To increase market profitability, we combine the features of token value, business applications and incentive mechanisms in one paradigm to propose a novel paradigm "dual incentive value-based paradigm" as the fourth paradigm in the business blockchain token economy. Different with the previous paradigms, the novel paradigm achieves dual incentive effects on different types of nodes by only one token to expend the usage scenario. Meanwhile, it contains token value design and the liquidation design. Therefore, the token can be used as real currency and the novel paradigm can be used in the business market economy.

To prove the applicability in the business scenario, we propose a study case for improving merchants' environment state in the business scenario. In this case, the government, the alliance, the merchant and the customer are four roles, and the voucher is the token. Then we set up two economic models to prove that the novel paradigm can be more profitable than the existing paradigms. After that, we make simulations to compare the expected profitability of merchants in non-paradigm, existing paradigm and novel paradigm scenarios. The result shows that merchants in the novel paradigm can obtain at most $10 \%$ more profit than those in the existing paradigm and at most $32 \%$ more profit than those without paradigms. The contributions of our study are as follows:

- We conclude three existing paradigms of the blockchain token economy novelly and analyze the shortcomings of each paradigm. 
- We propose a novel dual incentive value-based paradigm in the blockchain token economy. It can make dual incentive effects and enhance the entity value of the token in the business blockchain economy.

- We propose a study case and set up two economic models to prove the novel paradigm's profitability.

- We compare our novel paradigm with other paradigms in the study case to prove its effectiveness.

Our following sections are structured as follows: Section 2 summarizes existing paradigms of blockchain token economy; Section 3 proposes the novel "dual incentive value-based paradigm"; Section 4 proposes a business study case, a hierarchical alliance structure model to set the level for merchants and a new "newsvendor proble" profit model to evaluate the profit; Section 5 makes experiments to prove that the new paradigm is more profitable than the existing three paradigms and makes discussion and suggestions based on experiment results; Section 6 concludes our paper.

\section{Literature Review}

Blockchain token is originated from Bitcoin. At the early age of the blockchain development, the studies of the tokens are about the transcations of ICOs, such as the Bitcoin and the Ethereum. The ICO is a method of generating tokens and raising funds. It can be traded and liquidated as the currency. In February 2018, the Swiss Financial Market Supervisory Authority (FINMA) released ICO guidelines [23]. However, with the development of the ICO, more and more financial and economic problems are appearing, such as the scams and the fraud. As a consequence, the design of the Bitcoin is only used to stimulate nodes. Therefore, researchers design new tokens with incentive mechanisms in their customized business blockchain. This is the origin of the blockchain token economy.

Currently, researchers conduct blockchain token economy studies on three aspects: (1) the design of token's incentive mechanisms; (2) the design of particular economic scenarios; and (3) the relationship analysis between the ICO and the tokens.

The first aspect is the design of token's mechanisms. These studies on the blockchain token economy concentrate on how to design a token in detail and how to make token valuable. Since the incentive mechanism determines the rule of obtaining the tokens in the blockchain, some studies focus on designing novel incentive mechanisms. In these studies, a well-designed incentive mechanism can make nodes obtain tokens equally. It should also be able to control the circulation and balance the value of the token. Jung et al. [24] set up three roles in the healthcare blockchain and two real-world business scenarios of healthcare companies recruiting. In the scenarios, two economic models are set up by the game theory to explain the novel incentive mechanism for controlling the token's reward and the recruitment costs. The result shows the novel incentive mechanism can control them effectively. Drasch et al. [17] modeled a novel associated incentives on the token in a business blockchain platform. Drasch et al. proposed a two-stage incentive at the stage of platform development and platform operation. They use economic theory to analyze that tokens can help to overcome the "chicken and egg" problem and lead to contradictory incentives for platform participants. Takemiya [25] proposed a self-regulating token-based economic system and a dual-token system with a novel incentive mechanism. The selfregulating system is implemented through allocating votes in a Sybil-resistant manner. The dual-token system is designed in economic theories. In the incentive mechanism, each token's users can obtain the other token by storing and servicing their tokens. This could guarantee the users' and the system's benefit.

The second aspect is the design of particular economy scenarios. Generally, the economy contains sub-classifications, such as the circular economy, the market economy, and the government economy. In the blockchain token economy, different economies increase the variety of token value. In the service of different blockchains, different tokens can take different methods for nodes to obtain tokens. Therefore, researchers made studies on new economic scenarios to increase token variety in recent years. Kim et al. [19] 
proposed a process for building a desirable model of a token economy. It can make a sustainable growth. The sustainable growth of the token economy is implemented by voluntary activities of participants and well defined economic models. It can increase the participants' interests and obtain sustainable profit growth. Kim et al. set up the economic model by strategic managements and set up incentive mechanisms to support the economic model on the token for a sustainable growing token economy. Narayan et al. [18] proposed a tokenizing coopetition in the circular token economy. The circular economy offers a way for businesses to conceptualize sustainable economic activity with a concern for environmental and societal wellbeing. Narayan et al. set up circular models by using competition to operationalize and optimize using tokens.

The third aspect is the relationship analysis between the ICO and the tokens. Except the well-defined tokens in different business scenarios, ICO is also a type of token in the blockchain. Therefore, a small number of researchers study the relationship between ICO and the token. Tonnissen et al. [21] analyzed 195 ICOs and performed cluster-analysis in order to identify three different archetypes. The archetypes are the pioneering model, the expansion model and the authority model. They can offer research concerns on token-based business models.

After analyzing the studies, the research of three aspects can be summarized into three well-designed types: First, most of the studies on designing the incentive mechanism set up economic models or theories to design a new incentive mechanism in the business scenario; Second, most of the studies on the novel economic sub-classifications explain the sub-classification. They use non-economic methods to model the sub-classification, and use economic model to design the token in non-business scenario. Third, most of the studies on the relationship between the ICO and the token introduce the combination of ICOs and tokens without incentive mechanisms in the business scenario. Based on this, we summarize the existing blockchain token economy literature into three paradigms. The paradigm characteristics and disadvantages are as follows:

\section{(1) Incentive-based paradigm:}

The incentive-based paradigm concentrates on the design of the incentive mechanism of the token and how to attain the best situation of maximum incentive effect:

- Incentive Mechanism: It aims to make the platform obtain maximum benefits or make consumers cost minimum;

- Token Design: It includes guidelines for the issuance, trading and functioning of tokens;

- Token Visualization: It contains a flow chart or a sequence diagram to explain how the token works;

- Mechanism Feasibility Validation: It includes theoretical and experimental verification.

- Practical Application: A healthcare blockchain system [24]. It models an incentive mechanism of blockchain based on game theory and a mechanism design that recruits participants through a health care blockchain platform in game theory. It proposes three roles: participants, data recipients (companies) and data providers (health care organizations). It also defines rules how roles can obtain tokens and represents a figure to show the token visualization. Finally, it sets simulations to estimate the benefit which the incentive mechanism can provide for users and the cost for the company. Results show that the incentive mechanism can minimize the cost of the company and stimulate users to join.

However, two disadvantages exist in this paradigm:

- Disadvantage 1: Few literature defines multiply roles. The current paradigm only includes two roles such as the community and the participates (nodes). Therefore, only the single incentive mechanism exist between the community and the participates.

- Disadvantage 2: No literature introduces the relationship between its token and ICO or the currency. Therefore, the value of the token is virtual and can only be expressed in their design. 


\section{(2) New scenario-based paradigm:}

The new scenario-based paradigm concentrates on how the token economy works in a particular blockchain economic scenario, such as the circular economy and sustainable economy:

- Scenario Explanation: It explains the new scenario and the potential advantages of token economy use in non-traditional scenarios;

- Token Design: It includes the effect of the token in their new scenario.

- Theoretical model: Models for the new scenario that comes from any research field, such as the collaborative, crypto-economic, and management models;

- Research framework/flow: It includes two types: one does not contain the experiment based on the theories; the other contains the experiment with the research flow same as the incentive-based one.

- Practical Application: A blogging and social networking website "Steemit". It proposes a sustainable growing token economy [19]. It sets up a strategic model using cryptoeconomics and strategic management theories, which contains eight processes to define the token. It shows the research flow by a sequence diagram and theoretical analysis.

The disadvantage for the paradigm is shown as follows:

- Disadvantage: No literature applies the paradigm in the business scenario, and no literature defines roles. In a business scenario, multiple roles can be existing at the same time. Therefore, the paradigm lacks the regulation and control effect on the business market economy.

\section{(3) Business schema-based paradigm:}

The business schema-based paradigm introduces the application of tokens in the actual business schema, such as the joint application of ICO and tokens. It also optimizes the existing token economy system from the perspective of business economics and strengthens the value of its token. Moreover, the relationship between suppliers and consumers in the blockchain token economy is essential and needs to be considered:

- Supplier analysis: Supplier is the most critical role. It defines token issuing rules and using rules. This part contains the token's design and value and basic information of the supplier;

- Roles in the business schema;

- Practical Application: A blockchain-based token business start-up ecosystem [21]. It contains three roles: supplier, the network and participant. Suppliers work together in order to define the value proposition for each participant. The ecosystem uses theoretical analysis to show token visualization and mechanism feasibility.

Token visualization and Mechanism Feasibility Validation are the same with the incentive-based paradigm. Business schema-based paradigm's disadvantage is shown as follow.

- Disadvantage: Although the tokens in the paradigm can be circulated as a currency, no literature introduces the incentive mechanism. The value of the token in the paradigm may gradually bubble, such as virtual token fraud.

In summary, existing paradigms lack multiple incentive mechanisms, business applications and the liquidity of tokens. The design of incentive mechanisms and the valuable token determines consumers' consumer willingness and the profitability of the entire business market. Therefore, a novel paradigm with the multiple incentive mechanisms of a valuable token is essential to improve the business market profit in the blockchain token economy. 


\section{Dual Incentive Value-Based Paradigm}

In this paper, we propose a novel blockchain token economy paradigm called "Dual Incentive Value-based paradigm"(DIV for short). To solve the disadvantages in the existing paradigms and improve the profitability of the paradigm, the following parts are novelly designed in DIV.

(1) Dual incentive effects by a token: Among different roles of the market, there are dual incentive mechanisms. For example, the community stimulates suppliers, and suppliers stimulate consumers by offering tokens in different periods.

(2) Entity value of the token: The token has strong liquidity and can be traded with other real currency, such as the dollar.

(3) Liquidation of token: The token has a service period. The community will liquidate unused tokens at the end of each period.

(4) Hierarchical roles: Roles are hierarchical in DIV. Each role hierarchy can set up multiple roles, and roles in the same hierarchy have the same functions and permission.

(5) Business study case: DIV should contain a suitable business case, and the case needs to prove the profitability of DIV.

Thus DIV consists of four parts: Roles, token design, incentive mechanism design and paradigm execution flow.

Roles:

(1) Initiator: The initiator is the creator of the blockchain token economy applied in the paradigm. It is an authoritative organization or individual. It offers the authorization of the alliance to stimulate suppliers to join in.

(2) Alliance: The alliance is similar to the "league" in the business scenario. It accepts suppliers to join in. It also offers and liquidates the tokens to the members at each period. The alliance obtains members' membership fees at the beginning of each period and liquidates members' unused tokens at the end of each period. Moreover, an alliance can exist with more than one type of supplier simultaneously. For example, a shopping market league can hold different shops, such as snack shops and fruit shops.

(3) Supplier (Member): The supplier is similar to the "merchant" in the business scenario. If the supplier joins the alliance, it becomes a Member of the alliance and obtains tokens from it at beginning of each period. Suppliers cannot obtain tokens from the alliance if they do not join the alliance. Each member has a level that determines the number of tokens they can obtain from the alliance. The level is based on some pre-defined rules. Members offer their tokens to consumers to obtain more profits and consumers. Moreover, members should pay the membership fee regularly to the alliance.

(4) Consumer: The consumer is similar to the "participant" in the blockchain. It can obtain benefits from using tokens and taking part in members' activities more. Moreover, a supplier/member can hold more than one type of consumer simultaneously.

Token Design is as follows.

(1) Overview of the token: In the business market scenario, the token is similar to the "voucher". Members obtain tokens and offer tokens to consumers. Members should bear the discount of consumers' tokens, small profits but quick turnover. Moreover, members can obtain profit from token liquidation. The authority of the token is from the initiator, and the transactions of tokens are recorded on the blockchain.

(2) Token liquidation: In the business market scenario, to increase the liquidity of the token, the alliance liquidates the unused tokens of members and gives compensation to them based on their unused tokens numbers at the end of each period. In the blockchain, the liquidation of tokens means a signal block with unused tokens information, which is created and recorded on the blockchain.

(3) The representation of token's value: The token can be used directly as a currency. Therefore, it has strong liquidity and entity value than other tokens in the blockchain. Moreover, the token can benefit all the roles in the paradigm. Consumers obtain discounts from tokens. Members obtain more profit from offering tokens. The alliance obtains profit 
by obtaining membership fees from suppliers of the alliance. The circulation of tokens is shown in Figure 1.

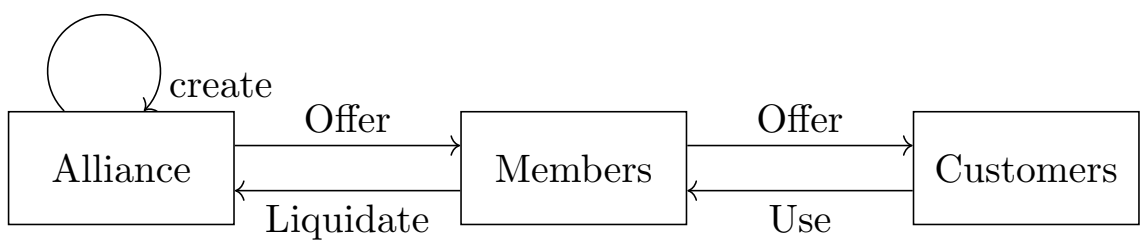

Figure 1. Circulation of tokens in the business-based paradigm. Tokens flow among the alliance, the members and the customers.

\section{Incentive mechanism design:}

In the paradigm, two types of incentive mechanisms coexist, and both use only one token.

- The incentive between the alliance and the members: The alliance sets levels and offers tokens to members. The profit of members increases by more consumers and liquidating from the alliance. The profit of members increases by more consumers and liquidating from the alliance.

- $\quad$ The incentive between the members and the consumers: Members offer tokens to consumers. Consumers obtain tokens and obtain discounts. Members need to bear the discounts of consumers, acquiring small profits but quick turnover.

Execution flow:

The total execution flow of the paradigm is shown in Figure 2.

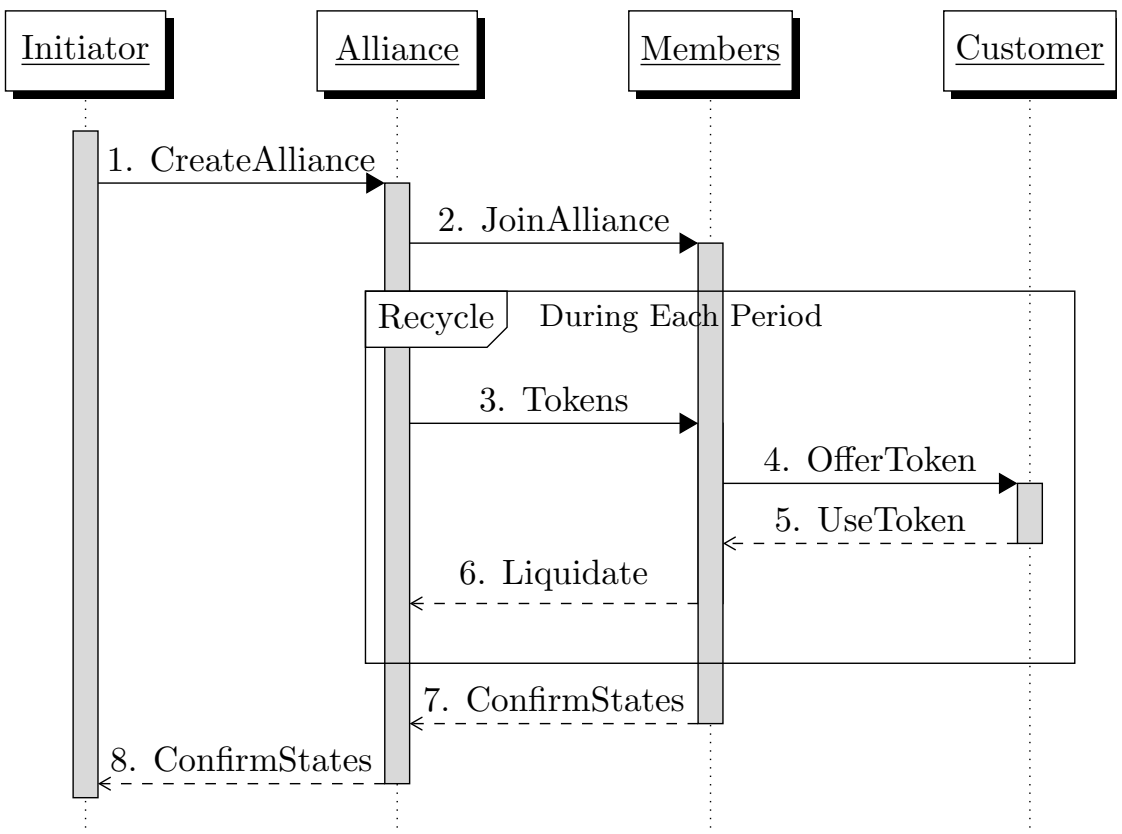

Figure 2. Total execution flow of the dual incentive value-based paradigm. The initiator creates the alliance. The alliance creates tokens and offers tokens to members at the beginning of a period. Members join in the alliance and offer tokens to customers. Customer can use tokens in members. When the period is over, the alliance liquidate all the unused token from members.

\section{Study Case: A Business Blockchain Alliance for Improving Environment and Profitability}

In this section, we propose a business blockchain alliance study case for improving environment and profitability (Env-profit for short) of DIV firstly. Then, we propose a 
theoretical analysis method to evaluate the profit of the roles in Env-profit. The route map is shown as Figure 3.

Value creation network: analyze the main factor in Env-profit.
Hierarchical alliance struc- New "Newsvendor probture model: set levels for $\longrightarrow$ lem" profit model: estimerchants mate profits.
Theoretical !

Analysis

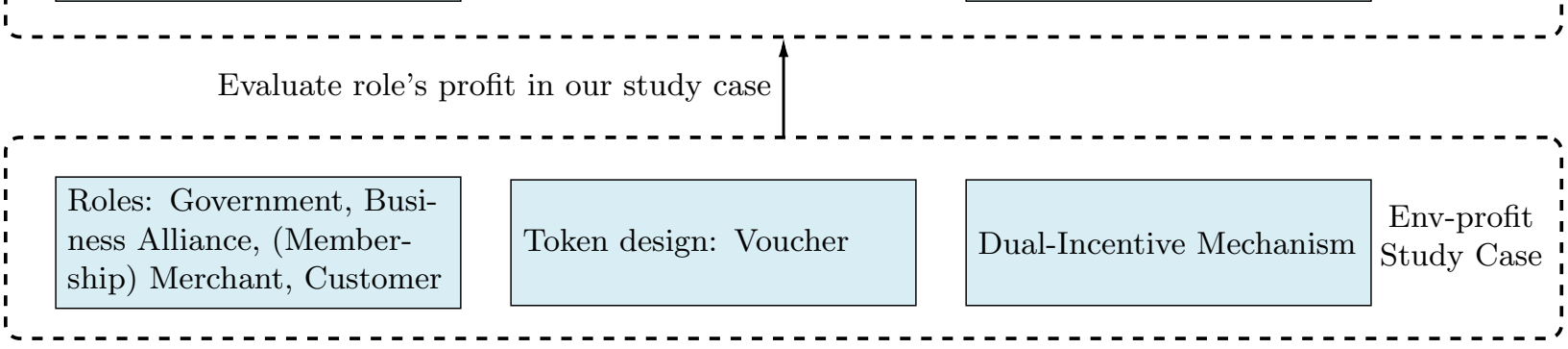

Figure 3. Route Map of the Study Case Env-Profit.

\subsection{Details of Env-Profit}

The corresponding relationships between Env-alliance and DIV are shown in Table 1.

Table 1. Corresponding relationships between the case and the paradigm.

\begin{tabular}{|c|c|c|}
\hline & DIV Paradigm & Env-Profit Case \\
\hline \multirow{4}{*}{ Roles } & Initiator & Government \\
\hline & Alliance & Business Alliance \\
\hline & Supplier/Member & Merchant/Membership Merchant \\
\hline & Consumer & Customer \\
\hline \multirow[b]{2}{*}{ Design } & Token's real value & Use as a currency and can be liquidated \\
\hline & Multiple incentive effects & $\begin{array}{l}\text { (1) Business alliance offers vouchers to membership merchants. } \\
\text { (2) Membership merchants offer vouchers to customers. }\end{array}$ \\
\hline Token & Token & Voucher \\
\hline
\end{tabular}

In Env-profitt, the government builds up a blockchain-based business alliance to stimulate merchants to improve air quality consciously. The business alliance is a decentralized and autonomous organization. It provides a membership system that each merchant can join the alliance. Each membership merchant has a level, which depends on the average air quality of a period. The membership merchant can obtain vouchers from the alliance as incentives based on its level. Moreover, each membership merchant should pay the membership fee to the alliance regularly.

The voucher is the token of the blockchain alliance. It has a use period, such as a month. It can be used as a currency for customers to obtain discounts. In Env-profit, the merchant can choose whether to join the alliance or not. If it joins, it can become a membership merchant. Moreover, only the membership merchant can obtain vouchers, and it should afford the discount of vouchers. Despite this, the alliance also offers a liquidation system to repurchase membership merchants' unused vouchers at the end of each period. Moreover, because of the authority of the alliance, customers can visit more membership merchants. In this manner, membership merchants obtain more customers and obtain more profits.

Customers are the users of vouchers, and they obtain vouchers from membership merchants. Moreover, the alliance gathers many high-air quality merchants so that customers will consume more in the league, which improves the profitability of the entire business market. The summary relationship of roles in Env-profit is shown in Figure 4. 
Finally, dual incentive effects exist in the Env-profit. The first incentive effect is that the alliance offers vouchers to membership merchants to improve air quality. The better air quality the membership merchants obtains, the higher the level they can be. The more vouchers they can have, the higher the profits they can have. The second incentive effect is that the membership merchants give out vouchers to customers to consume more, then membership merchants and the alliance can obtain more profits. Moreover, the voucher makes the token more valuable. It can be used as money, and its value and liquidity are shown.

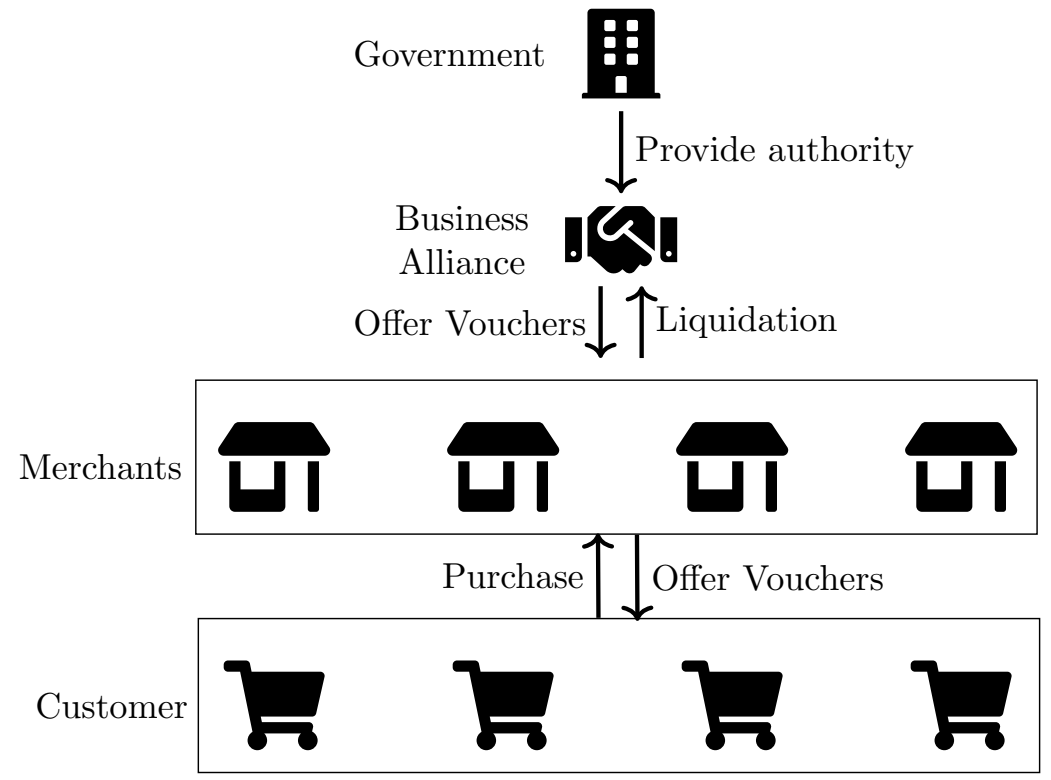

Figure 4. Summary relationship of roles in the case. Customers, merchants, the business alliance and the government are roles. The government provides the authority for the business alliance. The business alliance offer vouchers to merchants, and merchants offer vouchers to customers. Customers can purchase in merchants' by vouchers, and at the end of the period, the business alliance liquidates all unused vouchers from merchants.

\subsection{Theoretical Analysis}

In this section, we propose a theoretical analysis method to evaluate membership merchants' profit in Env-profit case. Firstly we set up a value creation network to analyze the main factor. Then, we propose two economic models: one is a hierarchical alliance structure model to set levels for merchants, and the other is a new "Newsvendor problem" profit model to evaluate membership merchants' profit.

\subsubsection{Value Creation Network}

We draw on the value net model concept, combine the value co-creation theory [26] and conclude a novel value creation network [27]. It consists of six creation modules: participants, external demand, value proposition, key business, core source and profit schema. The relationship among the modules is shown in the Figure 5. The corresponding relationships of the case is shown in the Table 2.

In Table 2, the level system effects the value proposition, key business and the core source modules, which mainly controls the entire paradigm. Therefore, we conclude that the level system is a significant model of the alliance; thus, we first set up the hierarchical alliance structure model to set levels of membership merchants. 
Table 2. Corresponding relationship of the case in the value creation model.

\begin{tabular}{cc}
\hline Value Creation Model Modules & Modules in the Case \\
\hline Participants & Merchants \\
\hline Value proposition & $\begin{array}{c}\text { Stimulate merchants by the level system and improve } \\
\text { the authority of external demand }\end{array}$ \\
\hline Key business & Set levels of merchants' air quality, give out and liquidate vouchers \\
\hline Core source & Voucher and authorize level system \\
\hline Profit schema & Collect membership fees
\end{tabular}

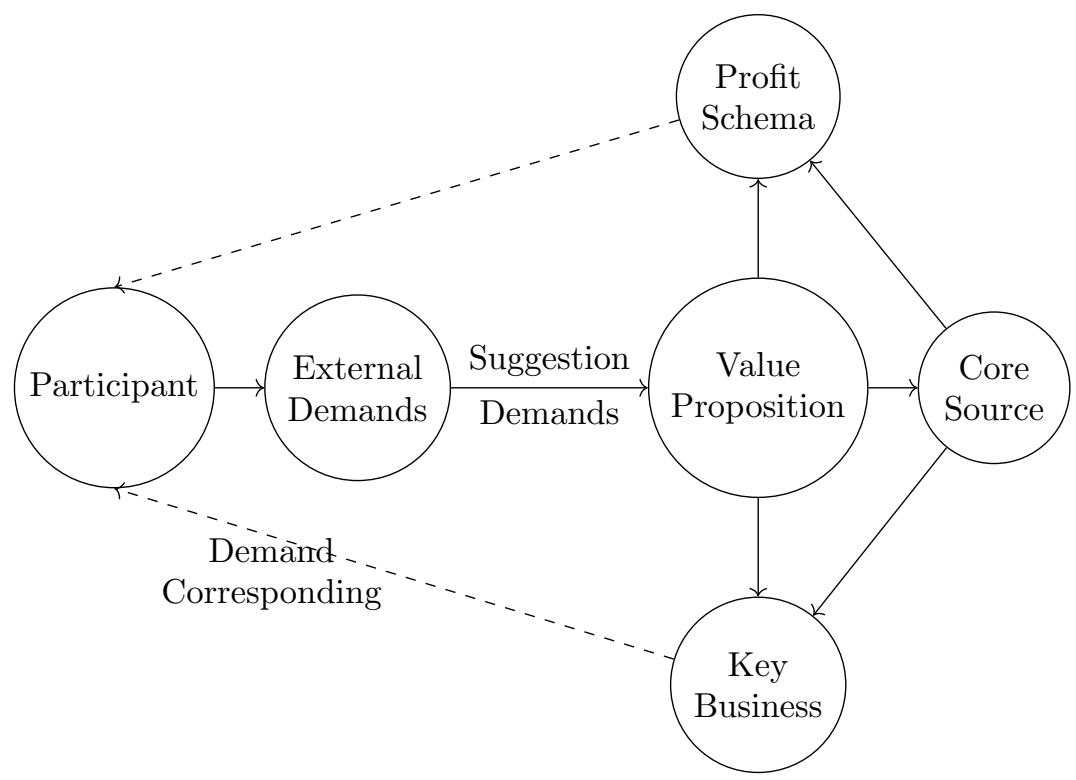

Figure 5. The relationship among value creation network model modules. The participant wants to create value. It has external demands. External demands can be exchanged into value proposition with receiving suggestions and demands. The value proposition is shown by the profit schema, the key business and the core source. The profit schema and the key business with demand correspondingly affect the participant's value, and the core source determines the profit schema and the business effect.

\subsubsection{Hierarchical Alliance Structure Model}

The effect of the merchant's level is shown in Figure 6, in which the membership merchant's score is transformed to the level. Three significant profit factors, namely the membership fee, the traffic and the number of vouchers, are affected by the level. This model sets up a rule to calculate membership merchants' scores and determine their levels in the alliance. The main steps are shown as follow:

1. We firstly need to find out all the corresponding qualitative and quantitative indicators;

2. Then, we set weights for each indicator;

3. Next, we calculate the scores based on the indicators' weights;

4. Later, we can set levels for membership merchants based on the score;

5. At last, we define other interactive functions in the Env-profit.

(1) Set indicators: We set qualitative and quantitative indicators of membership merchants that influence the level and is shown in Table 3. The quantitative factors are more important than qualitative factors. Therefore, we set the weight of quantitative indicators as $80 \%$ and the weight of qualitative indicators as $20 \%$.

(2) Set weights: We use the "Analytic Hierarchy Process" (AHP) [28] to confirm the weight of every single indicator. 
a. Construct the judgment matrix of indicators: The judgment matrix is denoted as $X_{\text {Level }} \in R^{n \times n}(n \in r)$, and $n=8$ in our case. $X_{\text {Level }}^{i, j}$ indicates the degree of importance between indicator $i$ and indicator $j$. The degree is shown in Table 4. Elements should follow rule $X_{\text {Level }}^{i, j} \times X_{\text {Level }}^{j, i}=1$. The final judgment matrix $X_{\text {Level }}$ is shown as Equation (1).

$$
X_{\text {Level }}=\left(\begin{array}{cccccccc}
1 & 1 & 2 & 2 & 4 & 4 & 3 & 5 \\
1 & 1 & 2 & 2 & 4 & 4 & 3 & 5 \\
\frac{1}{2} & \frac{1}{2} & 1 & 1 & 2 & 2 & 2 & 3 \\
\frac{1}{2} & \frac{1}{2} & 1 & 1 & 2 & 2 & 2 & 3 \\
\frac{1}{4} & \frac{1}{4} & \frac{1}{2} & \frac{1}{2} & 1 & 1 & 1 & 2 \\
\frac{1}{4} & \frac{1}{4} & \frac{1}{2} & \frac{1}{2} & 1 & 1 & 1 & 2 \\
\frac{1}{3} & \frac{1}{3} & \frac{1}{2} & \frac{1}{2} & 1 & 1 & 1 & 2 \\
\frac{1}{5} & \frac{1}{5} & \frac{1}{3} & \frac{1}{3} & \frac{1}{2} & \frac{1}{2} & \frac{1}{2} & 1
\end{array}\right)
$$

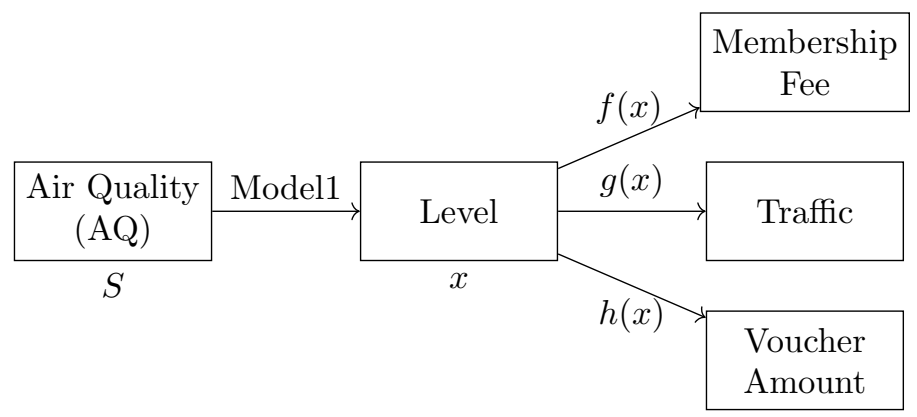

Figure 6. Importance of the merchant's level. The membership merchants' air quality(shorten as AQ) $S$ can be exchanged into a level $x$ by the hierarchical alliance structure model. Level $x$ can be exchanged to three key factors: the membership fee, the traffic of the membership merchant and the voucher amount of the membership. The corresponding functions between the level and three factors are defined as $f(x), g(x)$ and $h(x)$.

Table 3. Quantitative and qualitative indicators in the model.

\begin{tabular}{ccc}
\hline Indicator Type & Indicator Name & Symbol \\
\hline \multirow{3}{*}{ Qualitative } & Average number of used vouchers & $x_{1}$ \\
\cline { 2 - 3 } & Average air quality & $x_{2}$ \\
\cline { 2 - 3 } & Average number of customers & $x_{3}$ \\
\cline { 2 - 3 } & Average number of membership merchants' store visits & $x_{4}$ \\
\hline \multirow{2}{*}{ Quantitative } & Type of membership merchant & $x_{6}$ \\
\cline { 2 - 3 } & Merchant awareness & $x_{7}$ \\
\cline { 2 - 3 } & Reviews of membership merchant & $x_{8}$ \\
\hline
\end{tabular}

b. Use root method to calculate indicator weight: We use the root method to calculate each indicator's weight as $\overline{w_{i}}=\sqrt[n]{\sum_{j=1}^{n} x_{\text {Level }}^{i, j}}$. Then, we normalize the element in $W=\left(\overline{w_{1}}, \overline{w_{2}}, \overline{w_{3}}, \cdots, \overline{w_{8}}\right)$ as $w_{i}=\frac{\overline{w_{j}}}{\sum_{j=1}^{8} \overline{w_{j}}}$ and obtain the indicator weight vector $W_{\text {Level }}$ of $X_{\text {Level }}$ as Equation (2).

$$
\begin{aligned}
W_{\text {Level }} & =\left(w_{1}, w_{2}, w_{3}, \cdots, w_{8}\right) \\
& =(0.246,0.246,0.13,0.13,0.067,0.067,0.073,0.041)
\end{aligned}
$$


Table 4. Degree explanation of the elements.

\begin{tabular}{cc}
\hline Degree of $X_{i, j}$ & Explanation \\
\hline 1 & The $i$ th indicator is the same important with the $j$ th indicator. \\
\hline 3 & The $i$ th indicator is more important than the $j$ th indicator. \\
\hline 5 & The $i$ th indicator is strongly more important than the $j$ th indicator. \\
\hline 7 & The $i$ th indicator is absolutely more important than the $j$ th indicator. \\
\hline $2,4,6$ & Judgment median \\
\hline
\end{tabular}

c. Consistency test: We define the consistency indicator as $C(I)$, the average random indicator as $R(I)$ and the consistency ratio as $C(R)$. The corresponding relationship between $n$ and $R(I)$ is shown in Table 5, and Equation (3) shows the calculation of $C(R)$. If $C(R) \leq 0.1$, the judgment matrix $X_{\text {Level }}$ passes the consistency test. $C(I)=0.05$, $R(I)_{n=8}=1.41, C(R)=0.035<0.1$ and $W_{\text {Level }}$ pass the test. Thus, the weight of indicators is $W_{\text {Level }}$.

$$
C(R)=\frac{C(I)}{R(I)}=\frac{\left|\frac{1}{n} \sum_{i=1}^{n} \frac{\sum_{j=1}^{n} W_{L e v e l}^{i, j} \cdot w_{j}}{w_{i}}-n\right|}{(n-1) \cdot R(I)}
$$

Table 5. Relationship between indicator amount and R.I.

\begin{tabular}{ccccccccccc}
\hline $\mathbf{n}$ & 1 & 2 & 3 & 4 & 5 & 6 & 7 & 8 & 9 & 10 \\
\hline R.I. & 0 & 0 & 0.52 & 0.89 & 1.12 & 1.26 & 1.36 & 1.41 & 1.46 & 1.49 \\
\hline
\end{tabular}

(3) Calculate scores: This step contains a quantitative judgment model and a qualitative model to calculate the final score for membership merchants and set the level. In the quantitative judgment model, three parts are needed:

a. Confirm the quantitative indicators and judgment set: The quantitative indicators set is $\operatorname{Set}_{\mathrm{Quan}}=\left\{x_{1}, x_{2}, x_{3}, x_{4}, x_{5}\right\}$. We define the quantitative judgment set as $Y=\{$ Excellent, Good, Medium, Pass, Fail $\}$, and the max rating score is 100 . Based on the $80 \%$ weighting, the reference table between the score and the quantitative judgment set is in the Table 6.

$$
r_{i j}=\left\{\begin{array}{cc}
0 & \left(U_{i}<u_{i, j-1}, U_{i}<u_{i, j+1}\right) \\
\frac{u_{i}-u_{i, j-1}}{u_{i, j-1}-u_{i j}} & \left(u_{i, j-1}<U_{i}<u_{i j}\right) \\
\frac{u_{i, j}-U_{i}}{u_{i j}-u_{i, j+1}} & \left(u_{i j}<U_{i}<u_{i, j+1}\right)
\end{array}\right.
$$

Table 6. Judgment-Score reference table.

\begin{tabular}{cccccc}
\hline Judgment & Excellent & Good & Medium & Pass & Fail \\
\hline Score & $(65,80)$ & $(49,64)$ & $(33,48)$ & $(17,32)$ & $(1,16)$ \\
\hline
\end{tabular}

b. Confirm the indicator fuzzy judgment matrix: The judgment-indicator table is shown in Table 7, and each quantitative indicator owns a judgment score $p_{i}$. We define the indicator scoring vector $P_{Q u a n}=\left\{p_{1}, p_{2}, p_{3}, p_{4}, p_{5}\right\}^{T}$ and the indicator fuzzy judgment matrix $R_{Q u a n}=\left(r_{i, j}\right)_{5 \times 5} . r_{i j}$ means the membership degree of grade $j$ th judgment for the $i$ th indicator. Then, we define $U_{i}$ as the real value of each indicator, and $u_{i j}$ as the value border of each judgment for each indicator. The relationship among $r_{i j}, u_{i j}$ and $U_{i}$ is in 
Equation (4). For example, the real value of indicator $x_{1}$ is 450; thus, the $U_{i}$ of $x_{1}$ is 450 , according to the Table 7; the score $p_{1}$ is 64 ; and $u_{11}$ is $500, u_{12}$ is $300, r_{11}$ is 0.25 and $r_{12}$ is 0.75 . Each merchant has an indicator judgment fuzzy matrix $R_{Q u a n}$ according to its situation.

Table 7. Judgment-indicator table.

\begin{tabular}{cccccc}
\hline Indicator & $\begin{array}{c}\text { Excellent } \\
\mathbf{( 6 5 , 8 0 )}\end{array}$ & $\begin{array}{c}\text { Good } \\
\mathbf{( 4 9 , 6 4 )}\end{array}$ & $\begin{array}{c}\text { Medium } \\
\mathbf{( 3 3 , 4 8 )}\end{array}$ & $\begin{array}{c}\text { Pass } \\
\mathbf{( 1 7 , 3 2 )}\end{array}$ & $\begin{array}{c}\text { Fail } \\
\mathbf{( 0 ,} \mathbf{1 6})\end{array}$ \\
\hline$x_{1}$ (amount) & $>500$ & {$[300,500]$} & {$[150,300]$} & {$[50,150]$} & $<50$ \\
\hline$x_{2}$ (AQI) & $<40$ & {$[40,60]$} & {$[60,90]$} & {$[90,150]$} & $>150$ \\
\hline$x_{3}$ (amount) & $>1000$ & {$[500,1000]$} & {$[200,500]$} & {$[50,200]$} & $<50$ \\
\hline$x_{4}$ (amount) & $>750$ & {$[450,750]$} & {$[200,450]$} & {$[75,200]$} & $<75$ \\
\hline$x_{5}$ (amount) & $>3000$ & {$[1500,3000]$} & {$[500,1500]$} & {$[100,500]$} & $<100$ \\
\hline
\end{tabular}

c. Calculate the quantitative score with quantitative indicators weight: With the combination of $W_{Q u a n}, R_{Q u a n}$ and $P_{Q u a n}$, the final quantitative score $S$ core ${ }_{Q u a n}$ is as follows.

$$
\begin{aligned}
\text { Score }_{\text {Quan }} & =W_{\text {Quan }} \times R_{\text {Quan }} \times P_{\text {Quan }} \\
& =(0.246,0.246, \cdots, 0.067)\left(\begin{array}{lllll}
r_{11} & r_{12} & r_{13} & r_{14} & r_{15} \\
r_{21} & r_{22} & r_{23} & r_{24} & r_{25} \\
r_{31} & r_{32} & r_{33} & r_{34} & r_{35} \\
r_{41} & r_{42} & r_{43} & r_{44} & r_{45} \\
r_{51} & r_{52} & r_{53} & r_{54} & r_{55}
\end{array}\right)\left(p_{1}, p_{2}, p_{3}, p_{4}, p_{5}\right)^{T}
\end{aligned}
$$

In the qualitative judgment model, due to the uncertain value of qualitative indicators, the judgment of qualitative indicators should be signed manually. Each customer can give each merchant a score in $x_{7}, x_{8}$ and $x_{9}$ indicators and the total score of each indicator is 100. Due to the extreme scores, we calculate the average top $25 \%$ to $75 \%$ customers' scores of each indicator and obtain a customer score vector $C_{Q u a l}=\left(C_{x_{7}}, C_{x_{8}}, C_{x_{9}}\right)^{T}$. If a membership merchant has less than 50 customers' ratings, its qualitative score would be Score $_{\text {Qual }}=$ 0.25 Score $_{\text {Quan }}$. Based on the weights in $W_{\text {Level }}$, the weight vector of qualitative indicators $W_{Q u a l}$ is $(0.067,0.073,0.041)$, and the qualitative score is Score $_{\text {Qual }}=C_{\text {Qual }} \times W_{\text {Qual }}$. Thus, the total score of a membership merchant is Score $_{\text {Final }}=0.2$ Score $_{\mathrm{Qual}}+0.8$ Score $_{\mathrm{Quan}}$.

(4) Set levels: In Env-profit, we set that only the merchant's air quality is better than 70 and the merchant can enter the alliance. We set a score ranking-level table for membership merchants in Table 8. The hierarchical alliance structure model offers researchers reference on setting levels for an alliance.

Table 8. Ranking-Level table.

\begin{tabular}{cccccc}
\hline Ranking & Top 5\% & Top 5\% to 15\% & Top 15\% to 35\% & Top 35\% to 65\% & Bottom 35\% \\
\hline Level & 5 & 4 & 3 & 2 & 1
\end{tabular}

(5) Definitions of othe interactive functions in the Env-profit: We define some basic functions of the Env-profit. Based on Figure 6, we define the membership fee of merchants as $\mathrm{Fee}_{\mathrm{Mer}}\left(\right.$ Level $\left._{\mathrm{Mer}}\right)$, the traffic of merchants as $\operatorname{Traff} f_{\mathrm{Mer}}\left(\right.$ Level $\left._{M e r}\right)$ and the number of vouchers for merchants as Voucher $_{M e r}\left(\right.$ Level $\left._{M e r}\right)$.

The traffic of merchants in the case is affected by merchants' levels and the incentive of vouchers. Agarwal and Qian [29] propose a study that MPC (Marginal Propensity to Consume) [30] of giving vouchers can rise $15 \%$ to $40 \%$. In Env-profit, we set that membership merchants of Level 1 can obtain a 15\% increase and membership merchants of Level 5 can obtain a $40 \%$ increase in traffic based on [29]. In addition, merchants cannot 
increase the traffic. We make a logarithmic fit on the function of Level-Traffic increase, and it is shown in Equation (6). We obtain the traffic increase rate of each level in Env-profit in Table 9.

$$
\operatorname{Traff}_{\text {Mer }}\left(\text { Level }_{M e r}\right)=0.236 \ln \left(\text { Level }_{\text {Mer }}+1.129\right)+0.971 \text { Level }_{\text {Mer }}=0,1,2,3,4,5
$$

Table 9. Relationship between traffic increase rate and level.

\begin{tabular}{ccccccc}
\hline Level & 0 & 1 & 2 & 3 & 4 & 5 \\
\hline $\begin{array}{c}\text { Traffic increase rate } \\
\text { (new traffic/old traffic) }\end{array}$ & 1 & 1.15 & 1.24 & 1.306 & 1.357 & 1.4 \\
\hline
\end{tabular}

\subsubsection{New "Newsvendor Problem" Profit Model}

We introduce an alliance and the level system in the DIV to promote customers' consumption and increase profits of membership merchants. Therefore, we need to propose a novel profit model to reasonably estimate profits of membership merchants. "Newsvendor problem" [31] is a typical single-cycle storage problem in operations research. In Env-profit, membership merchants are similar to the newsvendors, and vouchers are similar to the newspapers. Therefore, we propose a new "newsvendor problem" profit model based on Env-profit to calculate the membership merchants' profits. In the DIV, the number of the customers is uncontrolled and essential to the membership merchants' profits, and other factors such as the discount and repurchase rate are controllable. Therefore, the objective of the model is to find the number of customers when a merchant can make the most profit.

Problem Description: A membership merchant has $Q$ vouchers at the beginning of the period. During the period, $r$ customers have used the voucher in the membership merchant. At the end of the period, the alliance repurchased the unused vouchers as $u$ dollars per voucher.

Problem Denotation: We have the following denotations in this problem:

- $\quad$ The possible value of $r$ is denoted as $r_{j},(j=1,2, \ldots, n)$;

- $\quad$ The membership merchant's average cost is denoted as $c$;

- The average selling price is denoted as $p,(u<c<p)$;

- $\quad$ The membership fee of membership merchant is denoted as $f(x)$;

- the discount of a voucher is denoted as $d \%$, and it is given;

- The probability of $r$ is satisfied with $P\left(r_{j}\right): 0<P\left(r_{j}\right)<1, \sum_{r>r_{n}} P(r)=1$.

Problem Objective: The objective of the problem is to obtain the best value of $Q$ when the membership merchant can obtain the most profit.

Solving: We firstly calculate the expectation of membership merchant's normal profit without repurchasing $E(R(r))$ and the profit of repurchasing $E\left(R_{u}(r)\right)$. Then, we calculate the accounting profit expectation of a membership merchant $W(Q)$. Next, we divide $W(Q)$ into a constant and a new function $F(Q)$, and we use the differential to find the extreme of $F(Q)$. After our analysis, the extreme of $F(Q)$ is the best value of $Q$.

Set membership merchant's normal profit without repurchasing $R(r)$ as follows.

$$
R(r)=\left\{\begin{array}{l}
r>Q: p \cdot a \% \cdot Q+(r-Q) p \\
r \leq Q: p \cdot d \% \cdot Q
\end{array}\right.
$$

The expectation of membership merchant's normal profit without repurchasing $E(R(r))$ is the following.

$$
E(R(r))=\sum R(r) P(r)=p \cdot \sum_{r>Q}[r+Q(d \%-1)] P(r)+p \cdot d \% \sum_{r \leq Q} r P(r)
$$

Set the profit of repurchasing $R_{u}(r)$ as follows. 


$$
R_{u}= \begin{cases}r>Q: & 0 \\ r \leq Q: & (Q-r) u\end{cases}
$$

Moreover, the expectation of the profit of repurchasing $E\left(R_{u}(r)\right)$ is $E\left(R_{u}(r)\right)=$ $\sum R_{u}(r) P(r)=u \cdot \sum_{r \leq Q}(Q-r) P(r)$; thus, the total profit expectation for a membership merchant in a period is as follows.

$$
\sum\left[R(r)+R_{u}(r)\right] P(r)=\sum R(r) P(r)+\sum R_{u}(r) P(r)
$$

The explicit cost expectation of the membership merchant at each period is $c r+f(x)$; thus, the accounting profit expectation of a membership merchant $W(Q)$ is as follows.

$$
\begin{aligned}
W(Q) & =\sum\left[R(r)+R_{u}(r)\right] P(r)-c r-f(x) \\
& =p \cdot \sum_{r>Q}[r+Q \cdot(d \%-1)] P(r)+p \cdot d \% \cdot \sum_{r \leq Q} r P(r)+u \cdot \sum_{r \leq Q}(Q-r) P(r) \\
& =\left[p \cdot d \% \cdot \sum r P(r)-f(x)-c r\right]-(Q-r)\left[p(1-d \%) \sum_{r>Q} P(r)-u \cdot \sum_{r \leq Q} P(r)\right]
\end{aligned}
$$

Set $F(Q)=(Q-r)\left[p(1-d \%) \cdot \sum_{r>Q} P(r)-u \cdot P(r)\right]$, because $\sum r P(r)$ is the expectation of $r$, and $p, d \%, f(x), c$ and $r$ are all constant values. We set Cons $=p \cdot d \% \cdot \sum r P(r)-$ $f(x)-c r$; then, we obtain $W(Q)=$ Cons $-F(Q)$. The goal of the model is to find the maximum value of $W(Q)$, and because of the finite number of values for $r$ and $Q$, the maximum of $W(Q)$ and the minimum of $F(Q)$ must be existing. Cons is a constant value; thus, the maximum value of $W(Q)$ is in the situation that $F(Q)$ is at the minimum value. To find the minimum of $F(Q)$, we set $F\left(r_{m}\right)=\min \left\{F\left(r_{j}\right) \mid j=1,2, \cdots, n\right\}(1 \leq m \leq n)$ and consider the differences of $\Delta F(Q)$ of $F\left(r_{m}\right)=F(Q)$. We set $r_{n+2}>r_{n+1}>r_{n}$; thus, the following is the case.

$$
\begin{aligned}
\Delta F(Q) & =\Delta F\left(r_{j}\right)=F\left(r_{j+1}-r_{j}\right) \\
& =\left(r_{j+1}-r_{j}\right)\left[p \cdot(1-d \%) \cdot \sum_{r>r_{j+1}} P(r)-u \cdot \sum_{r \leq r_{j}} P(r)\right]
\end{aligned}
$$

Using $\sum_{r>r_{j+1}} P(r)=1-\sum_{r \leq r_{j}} P(r), \Delta F(Q)=\left(r_{j+1}-r_{j}\right)[p \cdot(1-d \%)+u]$ $\left(\sum_{r>r_{j+1}} P(r)-\frac{u}{p \cdot\left(1-d^{\%}\right)+u}\right)$. Since $r_{j+1}>r_{j},[p \cdot(1-d \%)+u]>0$, when $\sum_{r>r_{j+1}} P(r)<$ $\frac{u}{p \cdot(1-d \%)+u}, \Delta F(Q)<0, F(Q)$ is decreasing, and when $\sum_{r>r_{j+1}} P(r)>\frac{u}{p \cdot\left(1-d^{\%} \%\right)+u}$, $\Delta F(Q)>0, F(Q)$ is increasing. Thus, when $\sum_{r>r_{j+1}} P(r)=\frac{u}{p \cdot(1-d \%)+u}, F(Q)$ has a minimum. Thus, the best value of $Q$ that can make the most profit is the following.

$$
Q=\left\{r \mid \sum_{r>r_{m+1}} P(r)=\frac{u}{p \cdot(1-d \%)+u}\right\}
$$

The final profit of membership merchant Profit $_{M e r}$ is the following.

$$
\text { Profit }_{\text {Mer }}=p \cdot d \% \cdot \sum r P(r)-c r-f(x)
$$

As long as the profit at $Q$ for membership merchants is more than the merchant's, the paradigm is usable and profitable. Thus, we make simulations to show the results of the model more accurately in Section 5.

\section{Experiment}

In this section, we make simulations to prove the profitability of DIV. Our codes for all experiments are available at https:/ / github.com/NEUSoftGreenAI/DIVEconomicBlockchain accessed on 28 January 2022. 


\subsection{Setup}

Environment: The hardware environment contains: an Intel Core i7-8750H CPU, 8G memory and a 240G hard disk. The software environment is as follows: Windows 10 and Jupyter Notebook under Python 3.8.

Simulations: We set up two simulations based on the Env-profit:

1. The first simulation proves membership merchants can obtain more profits than merchants.

2. The second simulation proves DIV is more profitable than the incentive-based, new scenario-based and business schema-based paradigm. The new scenario-based paradigm lacks business application, and Env-profit has solved the disadvantage. The business schema-based paradigm lacks the incentive mechanism; thus, it has no incentive. The incentive-based and the new scenario-based paradigms have a single incentive. Therefore, we compare merchants' profits among dual, single and no incentive scenarios. In the second simulation, we set up two single incentive scenarios based on the dual incentive paradigm:

- Air Quality Evaluation incentive (AQE for short): In the AQE scenario, the government directly offers tokens to stimulate merchants based on their air quality. No customers in the scenario. The government and the alliance are the same role. Merchants can obtain tokens to exchange money from the government.

- Merchants' Voucher incentive (MV for short) scenario: In the MV scenario, merchants directly offers vouchers to stimulate customers to consume more. No government and alliance in the scenario. Merchants need to bear the discount of vouchers and small profits, but it has quick turnover.

Assumptions: We have following assumptions in the experiments:

- Each period of Env-profit is a month. The merchants who are not in the alliance can obtain 1000 customers per month. The average sale price is $100 \$$ and the average cost is $50 \$$.

- The number of merchants' customers $r$ obeys standard normal distribution and the expectation is 1000 for merchants not in the alliance. For membership merchants, the expectation of $r$ is based on the level of merchants. The relationship between merchants' expectations and the level is shown in Figure 7.

- The discount of a voucher is a fixed rate to the selling price as $d \%$, and the repurchase money of each unused voucher is also a fixed rate to the selling price as $r p \%$. In the first simulation, we set $d \%=2.5 \%, 5 \%$ and $10 \%$ as a precondition.

- The variance of $r$ is $\sigma$, the expected number of customers for a merchant is Customer Exp $_{\text {, }}$ the z-score for $r$ is $z$ and the amount of voucher Voucher $_{M e r}$ that a membership merchant obtains is Voucher $_{\text {Mer }}\left(\right.$ Level $\left._{\text {Mer }}\right)=$ Customer $_{\text {Exp }}+\sigma \cdot z$.

Baseline The situation of merchants in the no incentive and dual incentive scenarios is the baseline. The baseline number of customers is 1000, the baseline profit of the alliance is $0 \$$ and the baseline profit of a merchant is $50,000 \$$.

Test Case Design: In the two simulations, we set up seven test cases as follows, and the result of each test case is analyzed from Sections 5.2.1-5.2.7:

Test Case 1: In the first simulation, when $d \%=5 \%$, we compare the profit in different $\sigma$ and $r p \%$ to analyze the impact of the number of customers on membership merchants' profits.

Test Case 2: In the first simulation, when $d \%=5 \%$, we analyze the relationship between $r p \%$ and $\sigma$ to obtain a suitable range of $\sigma$.

Test Case 3: In the first simulation, when $d \%=5 \%$, we estimate profit comparison for merchants of different levels and $r$ to prove that the profitability of DIV can increase as the level and $r$ increase.

Test Case 4: In the first simulation, when $d \%=5 \%$, we evaluate the expected profits for alliance and membership merchants to prove that both the alliance and the membership merchants can obtain more profits than the baseline. 
Test Case 5: In the first simulation, when $d \%=5 \%$, we estimate the expected profit of membership merchants in different levels and $r p \%$ to validate that the DIV's profitability is more than the baseline's roundly.

Test Case 6: In the first simulation, we compare the value of $\sigma$ when $r p \%=2.5 \%, 5 \%$, $10 \%$ to explain that the suitable value of $d \%$ is $5 \%$.

Test Case 7: In the second simulation, we compare alliance and merchant's expected profit at the same $d \%$ and $r p \%$ under four scenarios (no incentive mechanism, AQE, MV and Dual). We change the level of merchant to prove that the profitability of dual incentive scenario is better than other scenarios'.

Metrics: In the first simulation, based on the assumptions and baseline, if a customer uses the voucher, a membership merchant can earn $50-100 \cdot d \% \$$. If a voucher is repurchased, the merchant can obtain $100 \cdot r p \% \$$ profit. We set the actual number of customers for a merchant as Customer real, and the actual profit of a merchant in the alliance Profit real $_{\text {is }}$ the following.

$$
\text { Profit }_{\text {real }}=\left\{\begin{array}{l}
\text { Voucher }_{\text {Mer }}>\text { Customer }_{\text {real }}:(50-100 \cdot d \%) \text { Customer }_{\text {real }}+100\left(\text { Voucher }_{\text {Mer }}-\text { Customer }_{\text {real }}\right) \cdot \text { rp } \% \\
\text { Voucher }_{\text {Mer }} \leq \text { Customer }_{\text {real }}:(50-100 \cdot d \%) \text { Voucher }_{\text {Mer }}+50\left(\text { Customer }_{\text {real }}-\text { Voucher }_{\text {Mer }}\right)
\end{array}\right.
$$

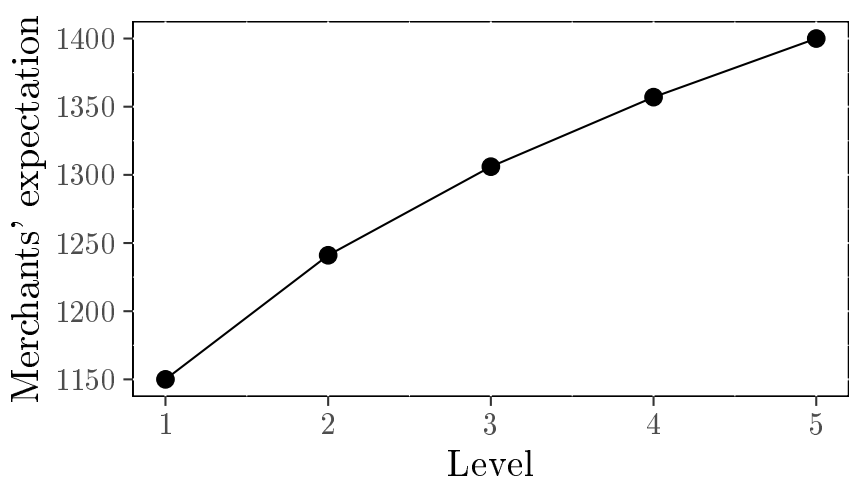

Figure 7. The relationship of merchants' expectation and level. The corresponding merchants' expectations from Level1 to Level5 are 1150, 1241, 1306, 1357 and 1400.

In Section 3, we experience the situation in which the merchant can obtain the maximum profit in Equation (13), as we assumed that the repurchased money $u$ is $100 \cdot r p \%$, and $p$ is 100 . Therefore, the formula can be simplified as follows.

$$
Q=\left\{r \mid \sum_{r>r_{m+1}} P(r)=\frac{100 \cdot r p \%}{100 \cdot(1-d \%)+100 \cdot r p \%}=\frac{r p \%}{1-d \%+r p \%}\right\}
$$

Since $\sum_{r>r_{m+1}} P(r)+\sum_{r \leq r_{m+1}} P(r)=1, \sum_{r \leq r_{m+1}} P(r)=\frac{1-d \%}{1-d \%+r p^{\%}}$, the z-score $z$ of $r$ can be obtained from the standard normal distribution table. The variance $\sigma$ of $r$ is calculated by the baseline situation.

$$
\sigma=\frac{\frac{50000-\text { Customer }_{\text {real }} \cdot(50-100 \cdot d \%)}{100 \cdot r p \%}-\text { Customer }_{\text {Exp }}}{z}
$$

In addition, the profit of the alliance is denoted as Profit $_{A l}$. It is the sum of the membership fees and minus the cost of repurchasing the vouchers. We set the membership fee as $\mathrm{Fee}_{\mathrm{Mer}}\left(\right.$ Level $\left._{\mathrm{Mer}}\right)=0.5 \cdot r \cdot$ Level $\quad($ Level $=1,2,3,4,5)$.

In the second simulation, we set the profit and the number of merchant's vouchers in the AQE scenario as Profit $A Q E$ and Voucher $_{A Q E}$, and those in the MV scenario as Profit ${ }_{M V}$ and Voucher $_{M V}$. In the AQE scenario, we assume that the profit of tokens for merchants is Profit $_{A Q E}=50,000+d \% \times$ Voucher $_{A Q E} \times 100$. We define Voucher $_{A Q E}=200$ Level $_{(\text {Level }}=$ $1,2,3,4,5)$. In the MV scenario, we set that the number of vouchers of a merchant obeys all assumptions. Therefore, the equation of Profit $_{M V}$ is as follows. 
Profit $_{M V}=\left\{\begin{array}{l}\text { Voucher }_{M V}>\text { Customer }_{\text {real }}:(50-100 \cdot d \%) \cdot \text { Customer }_{\text {real }} \\ \text { Voucher }_{M V} \leq \text { Customer }_{\text {real }}:(50-100 \cdot d \%) \cdot \text { Voucher }_{M V}+50\left(\text { Customer }_{\text {real }}-\text { Voucher }_{M V}\right)\end{array}\right.$

\subsection{Results}

In this section, we show the experiment results of the seven test cases above.

\subsubsection{Test Case 1: Result}

In the first situation, we set the default level as Level 1. When $d \%=5 \%$, Figure 8 shows the membership merchants' expected profit comparison when $\sigma$ is from 50 to 800 and $r p \%=10 \%, 15 \%$ and $20 \%$. It shows that when $\sigma$ increases, the membership merchant's profit also increases. Moreover, with the increase in $r p \%$, the membership merchant can reach the baseline at a minor $\sigma$. The larger $\sigma$ indicates that the number of customers is more unstable. The larger $r p \%$ indicates the profit of the alliance reduces. Therefore, a suitable $\sigma$ and $r p \%$ is critical for both alliance and membership merchants.

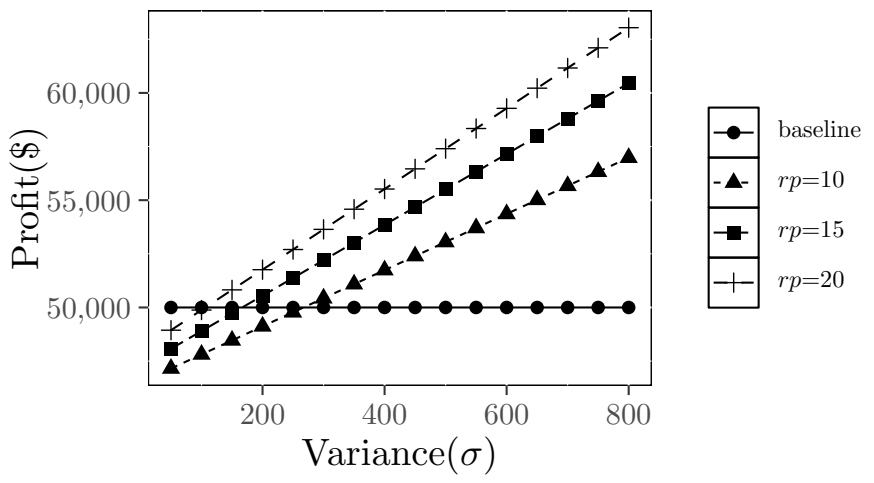

Figure 8. Profits comparison for different $\sigma$ and $r p \%$.

\subsubsection{Test Case 2: Result}

Figure 9 shows the relationship between $r p \%$ and $\sigma$ when $r p \%$ is from $5 \%$ to $20 \%$. It shows that when $r p \%$ rises, $\sigma$ decreases slower and slower. The expectation of $r$ for membership merchants in Level 1 is 1150. The membership merchant should ensure that the maximum fluctuation is not more than half of our expectations. It means $2 \sigma<\frac{1150}{2}=575$, $\sigma<287.5$; thus, $r p \%<10 \%$ is unsuitable. We set $r p \%$ as $10 \%$ to $20 \%$ in the following analysis.

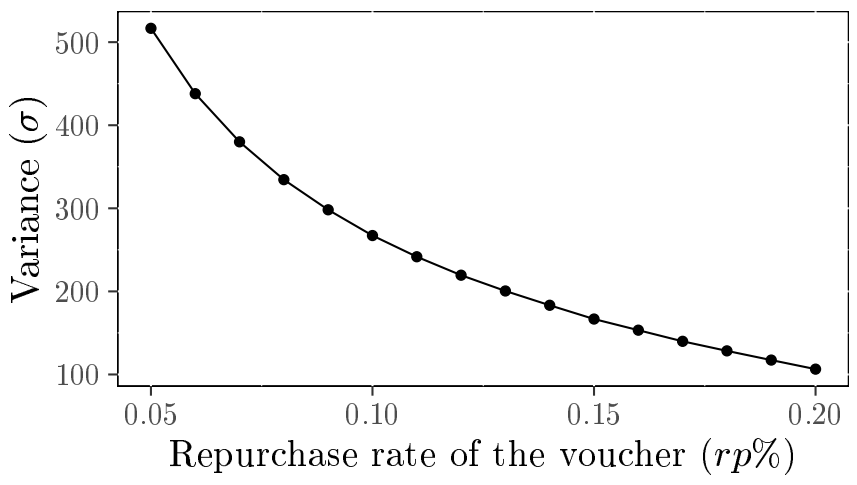

Figure 9. Relationship between $r p \%$ and $\sigma$. 


\subsubsection{Test Case 3: Result}

Figure 10 shows the membership merchants' expected profit in different levels when $r p \%$ is from $10 \%$ to $20 \%$. When the level rises, the profit of the same $r p \%$ also increases. Moreover, when the number of customers is the same as the baseline, membership merchants are also more profitable than the baseline. Figure 11 shows the relationship between $r$ and membership merchant's profit when $r p \%$ is $10 \%, 15 \%$ and $20 \%$. It shows that the profit increases in different $r p \%$ when $r$ increases. When $r>1000$, the profit is higher than the baseline in any $r p \%$. Moreover, we can conclude that each line in Figure 11 has a turning point. The bigger $r p \%$ is, the fewer vouchers merchant can obtain. The fewer profit merchants get, the earlier the turning point appears. Figures 10 and 11 validate the expected result that DIV is more profitable than the baseline when parameters are fixed at level-profit and $r$-profit.

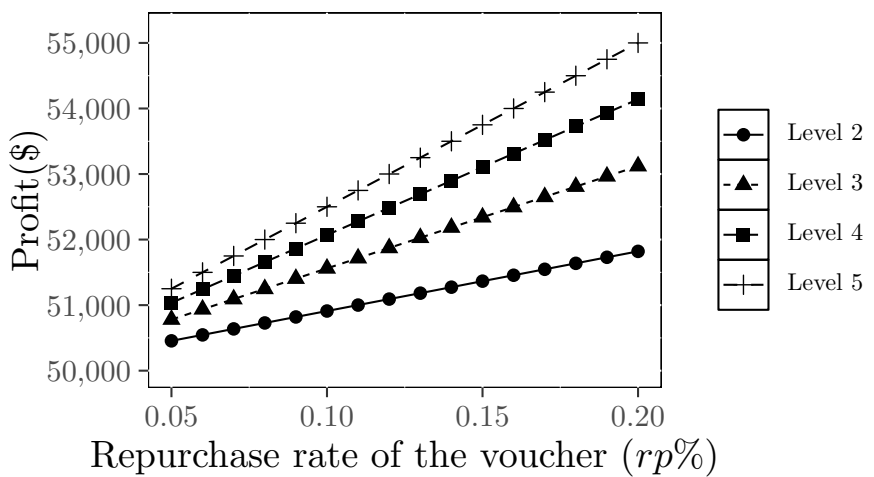

Figure 10. Merchants' profits comparison in levels and $r p \%$.

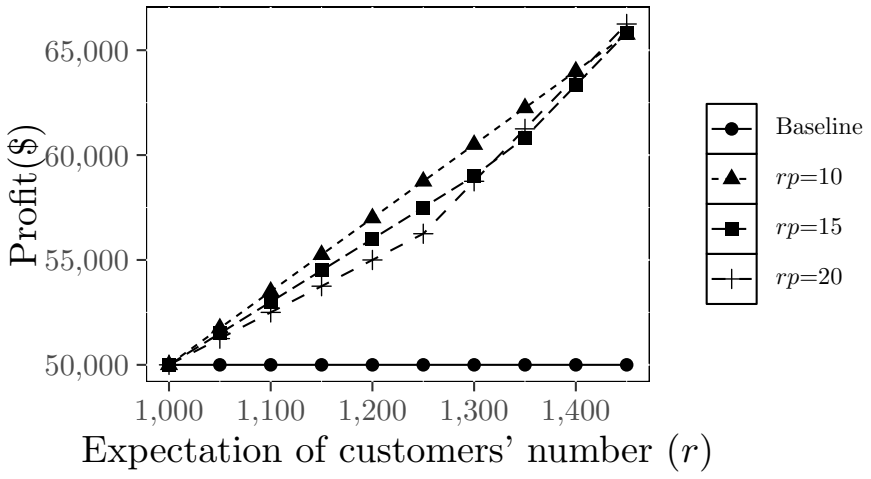

Figure 11. Profits comparison for merchants in $r$ and $r p \%$.

\subsubsection{Test Case 4: Result}

Figure 12 considers the alliance's profit and shows the expected profits for the alliance and membership merchants. The membership merchant's profit here is below the baseline of 50,000, and the alliance's baseline profit is 0 . We can conclude that membership merchants and the alliance can all obtain more profits than the baseline in expected situations. The higher the membership merchant's level is, the more profit the membership merchant and the alliance can obtain. 


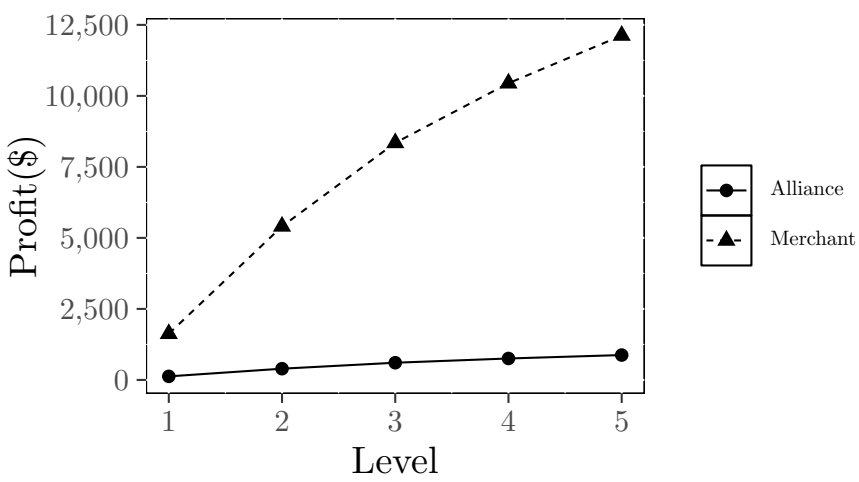

Figure 12. Expected profits for alliance and merchants.

\subsubsection{Test Case 5: Result}

Figure 13 shows the expected profit of merchants in each level (we set the baseline as Level 0) and expected $r$. It shows that the profit of membership merchants can be improved from $51,612.5$ to 66,000 . It is $3.2 \%$ to $32 \%$ more than merchants who are not in the alliance. In our case, all membership merchants can earn more profit. Therefore, the novel paradigm is more profitable than the baseline.

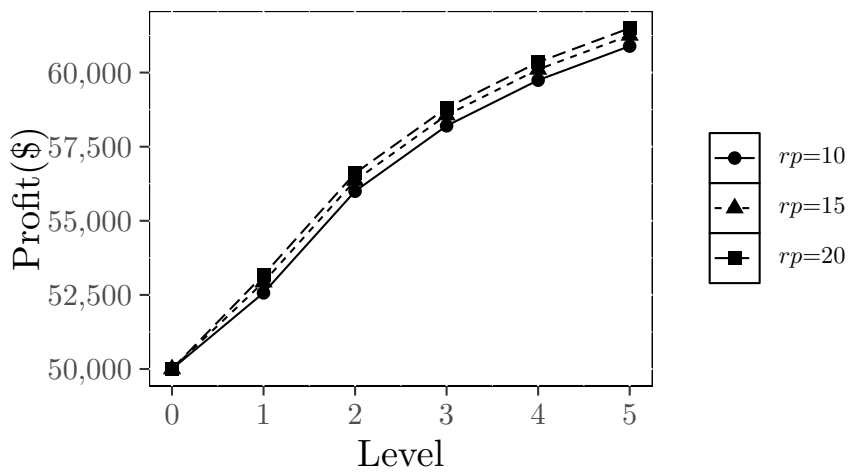

Figure 13. Expected profits of merchants in levels and $r p \%$.

\subsubsection{Test Case 6: Result}

Figure 14 represents the value of $\sigma$ when $r p \%$ is from $10 \%$ to $20 \%$ and $d \%$ is $2.5 \%, 5 \%$ and $10 \%$. It shows that when $d \%$ is too tiny $(d \%=2.5 \%), \sigma$ is very small even negative, which means that the discount of the voucher is too small and membership merchants do not use enough vouchers and even do not need vouchers to obtain the baseline profit. Therefore, we conclude that when $\sigma$ is too small, and the incentive mechanism is useless. Moreover, $\sigma$ is very large when $d \%$ is too large $(d \%=10 \%)$. It means that the discount of the voucher is so big that membership merchants need many vouchers to reach the baseline, and the fluctuation in the number of customers is large as well. However, we have analyzed that $\sigma$ is less than 287.5 in Section 5.2.2. When $d \%=10 \%$ and $r p \%=20 \%, \sigma$ is still more than 287.5. Therefore, the situation of $d \%=5 \%$ is more suitable than $d \%=2.5 \%$ and $d \%=10 \%$. A suitable $d \%$ is needed in DIV.

\subsubsection{Test Case 7: Result}

In the second simulation. We calculate the expected profit of each merchant and the alliance of four scenarios in different levels. Based on the first simulation, we set $d \%=5 \%$, $r p \%=10 \%$, and the value of $r$ is the expectation of different levels.

Figure 15 shows the alliance's profit from one merchant in four scenarios. As mentioned, there is no alliance in the MV scenario and no incentive scenario. Therefore, their alliance's profit is zero. The alliance's profit in the AQE scenario is negative. It decreases as 
the level increases, because the token profits of merchants in the AQE scenario is from the alliance. Therefore, the AQE scenario is not profitable for the alliance. It is not suitable in the business market either. The profit in the dual incentive scenario is positive. It increases as the level increases. Therefore, only the dual incentive scenario can make profits for the alliance. The dual incentive paradigm is friendly relative to large alliances and governments in the business market.

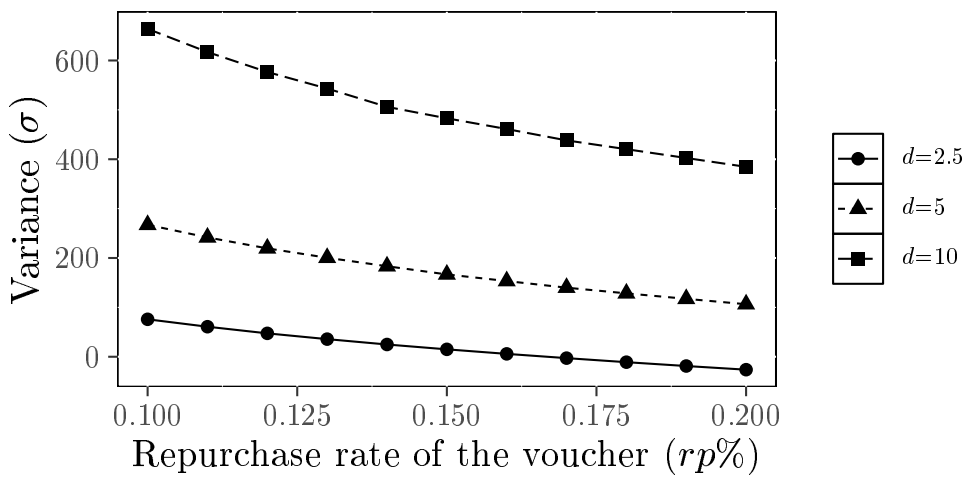

Figure 14. The value of $\sigma$ in different $r p \%$ and $d \%$.

Figure 16 shows the merchant's profit comparison of four scenarios in five levels. We can conclude that the profit of the AQE, MV and dual incentive scenario can increase as the level increases. Moreover, the profit of the dual incentive scenario is the highest in each level. The result is in line with our expectations.

To sum up, DIV is more profitable than the baseline and all existing blockchain token economy paradigms. DIV is the most suitable paradigm in business blockchain token economy scenario at present.

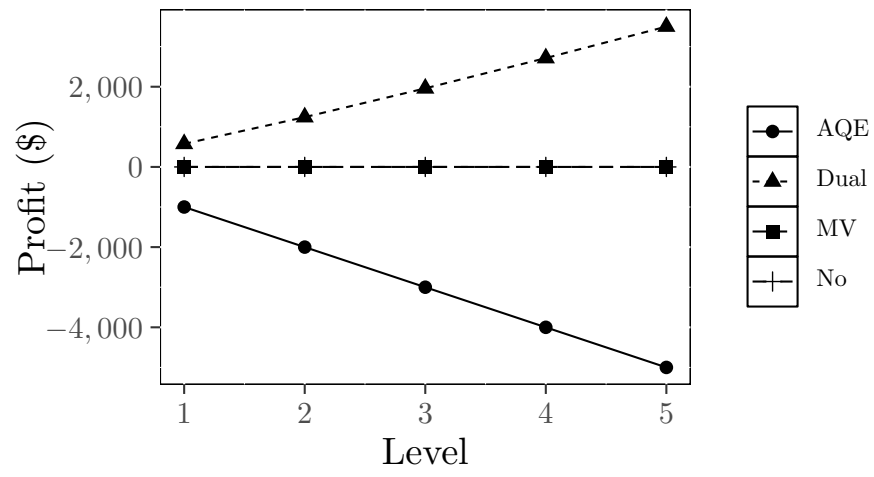

Figure 15. Alliance's profit comparison of four scenarios.

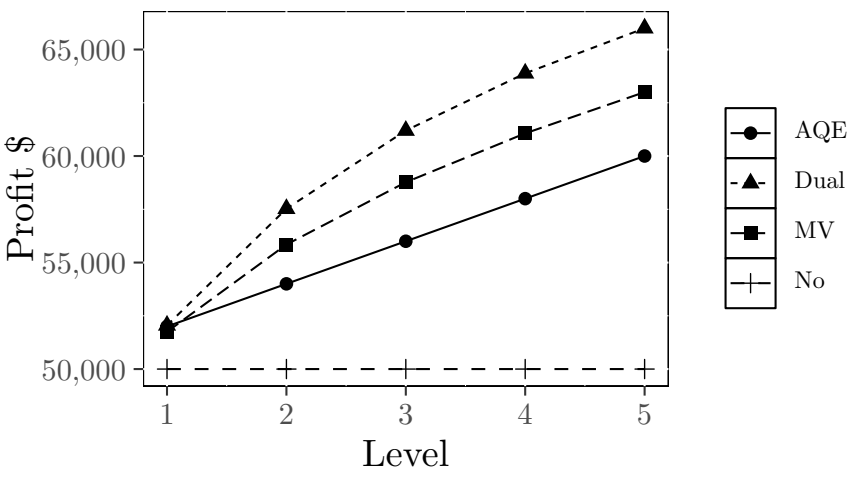

Figure 16. Merchant's profit comparison of four scenarios. 


\subsection{Discussion and Suggestions}

Based on the experiments, we can conclude that the DIV paradigm can be more profitable than other paradigms in the Env-profit case. In our analysis, the alliance and the customers are the two essential roles in the DIV paradigm. The alliance offers the second incentive mechanism to the merchants. The merchants receive the second incentive mechanism and offer more tokens to customers. Therefore, the customers can obtain more tokens and consume more and increase market profitability. There are two reasons why existing paradigms are less profitable than the DIV paradigm: (1) The business schemabased paradigms and the incentive-based incentive lack the role of alliance, which lack the second incentive mechanism. (2) The new scenario-based paradigm lacks business applications, which lacks dual incentive mechanisms in the business scenario. Overall, the DIV paradigm is the most profitable paradigm in our case. It implements the exchange between the currency and the token by suitable incentive mechanisms in the business scenarios. Moreover, it increases the liquidity and the value of the token.

Currently, people's enthusiasm on ICOs increased, but the value of ICOs is being criticized. We suggest that the ICO market can be applied the DIV in future. DIV can provide a complete set of ICO acquisition, ICO repurchase and the ICO transaction process. Therefore the value of the ICO is guaranteed and supervised by the DIV paradigm. The authority, liquidity and credibility of the ICO are also guaranteed. Moreover, we set up a business alliance in the Env-profit, and the business alliance can hold different merchants. We suggest that a blockchain token league can be founded in the real blockchain economy. Different tokens and ICOs can be traded and exchanged as currencies in the league. Moreover, the league can design novel cross-chain tokens and implement the cross-chain transactions of the tokens. However, the transformation rules of different ICOs and tokens and the suitable incentive mechanism to obtain different ICOs and tokens should be well designed.

\section{Conclusions}

The blockchain token economy has played an essential role in the business market. It increases the economy's security and strengthens the marketization of resource allocation. The incentive-based paradigm, the new scenario-based and the business schema-based paradigm are existing blockchain token economy paradigms. They lack the incentive mechanism, the business application and the token's liquidity. They can maximize the consumers' willingness to consume, but they can also decrease the profitability of the entire business market. In this paper, we propose a "Dual incentive value-based" paradigm (DIV), including roles, token design, incentive mechanism design and execution flow. Then, we propose a business study case called "Env-profit" based on the DIV paradigm. We propose a hierarchical alliance structure model and a new "newsvendor problem" profit model in Env-profit and set up two simulations to prove the profitability of DIV. The results show that the alliance of the DIV paradigm can make all the members be more profitable than those not in the alliance, and the DIV paradigm can be more profitable than other three existing paradigms. Based on the results, we analyze that the alliance provides the second incentive mechanism in order to stimulate customers to consume and profitability increases. Existing paradigms all lack the role of alliance so that profitability is less than the DIV paradigm without the second incentive mechanism. Overall, the DIV paradigm set the incentive mechanism to obtain tokens. The tokens can be liquidated, used and traded as real currencies. It increases the value of the token and market profitability. Moreover, the DIV paradigm will be widely used in the business blockchain, which can offers new research directions for researchers. For example, the ICO market can be applied the DIV, and a blockchain token league containing different tokens and ICOs can be founded in the real blockchain economy.

In addition to profitability, risk assessment is also an essential part of economic theory. In this paper, we focus on dual incentive mechanism research and do not consider the risk control mechanisms. In the future, if there are risks in the DIV environment, the methods for strengthening the risk avoidance ability of the DIV paradigm will be our novel research 
priorities. We believe that the DIV paradigm can be the leader in blockchain token economy study, and the blockchain token economy will be the mainstream in the token economy.

Author Contributions: Conceptualization, C.G., P.Z., B.L. and J.S.; data curation, C.G. and P.Z.; formal analysis, C.G., P.Z., B.L. and J.S.; investigation, B.L.; methodology, C.G., P.Z. and J.S.; project administration, C.G.; resources, C.G. and B.L.; validation, P.Z.; visualization, C.G., P.Z. and B.L.; writing-original draft, C.G. and P.Z.; writing-review and editing, C.G. and J.S. All authors have read and agreed to the published version of the manuscript.

Funding: This research was funded by the Natural Science Foundation of Liaoning Province (2020BS-054), the Fundamental Research Funds for the Central Universities (N2017005) and the National Natural Science Foundation of China (62162050).

Institutional Review Board Statement: Not applicable.

Informed Consent Statement: Not applicable.

Data Availability Statement: Data are contained within the article.

Conflicts of Interest: The authors declare no conflict of interest.

\author{
Abbreviations \\ The following abbreviations are used in this manuscript: \\ DIV Dual Incentive Value-based paradigm; \\ Env-profit a business blockchain alliance case for improving the environment and profitability; \\ AQE Air Quality Evaluation incentive scenario; \\ MV Merchants' Voucher incentive scenario.
}

\title{
References
}

1. Letychevsky, O.; Peschanenko, V.; Radchenko, V.; Poltoratzkyi, M.; Kovalenko, P.; Mogylko, S. Formal Verification of Token Economy Models. In Proceedings of the 2019 IEEE International Conference on Blockchain and Cryptocurrency (ICBC), Seoul, Korea, 14-17 May 2019; pp. 201-204. [CrossRef]

2. Wittek, K.; Lazzati, L.; Bothe, D.; Sinnaeve, A.J.; Pohlmann, N. An SSI Based System for Incentivized and SelfDetermined Customer-to-Business Data Sharing in a Local Economy Context. In Proceedings of the 2020 IEEE European Technology and Engineering Management Summit (E-TEMS), Dortmund, Germany, 5-7 March 2020; pp. 1-5. [CrossRef]

3. Jorgenson, D.W. Production and welfare: Progress in economic measurement. J. Econ. Lit. 2018, 56, 867-919. [CrossRef]

4. Aziz, N.A.A.; Yasin, M.H.M. Token Economy to Improve Concentration among Students with Learning Disabilities in Primary School. J. ICSAR 2018, 2, 32-36. [CrossRef]

5. Song, J.; Zhang, P.; Alkubati, M.; Bao, Y.; Yu, G. Research advances on blockchain-as-a-service: Architectures, applications and challenges. Digit. Commun. Netw. 2021. [CrossRef]

6. Zhang, P.; Song, J. Research Advance on Efficiency Optimization of Blockchain Consensus Algorithms. Comput. Sci. 2020, 47, 296-303.

7. Nanayakkara, S.; Rodrigo, M.; Perera, S.; Weerasuriya, G.; Hijazi, A.A. A methodology for selection of a Blockchain platform to develop an enterprise system. J. Ind. Inf. Integr. 2021, 23, 100215. [CrossRef]

8. Khan, A.A.; Shaikh, Z.A.; Baitenova, L.; Mutaliyeva, L.; Moiseev, N.; Mikhaylov, A.; Laghari, A.A.; Idris, S.A.; Alshazly, H. QoS-Ledger: Smart Contracts and Metaheuristic for Secure Quality-of-Service and Cost-Efficient Scheduling of Medical-Data Processing. Electronicsweek 2021, 10, 3083. [CrossRef]

9. Ayub Khan, A.; Laghari, A.A.; Shaikh, A.A.; Bourouis, S.; Mamlouk, A.M.; Alshazly, H. Educational Blockchain: A Secure Degree Attestation and Verification Traceability Architecture for Higher Education Commission. Appl. Sci. 2021, 11, 10917. [CrossRef]

10. Cao, B.; Xia, S.; Li, Y.; Li, B. An incentive-based workload assignment with power allocation in ad hoc cloud. In Proceedings of the 2017 IEEE International Conference on Communications (ICC), Paris, France, 21-25 May 2017; pp. 1-6. [CrossRef]

11. Xia, S.; Yao, Z.; Li, Y.; Mao, S. Online Distributed Offloading and Computing Resource Management with Energy Harvesting for Heterogeneous MEC-Enabled IoT. IEEE Trans. Wirel. Commun. 2021, 20, 6743-6757. [CrossRef]

12. Kim, H.M.; Laskowski, M.; Zargham, M.; Turesson, H.; Barlin, M.; Kabanov, D. Token Economics in Real Life: Cryptocurrency and Incentives Design for Insolar's Blockchain Network. Computer 2021, 54, 70-80. [CrossRef]

13. Lee, J.Y. A decentralized token economy: How blockchain and cryptocurrency can revolutionize business. Bus. Horizons 2019, 62, 773-784. [CrossRef]

14. Ivy, J.W.; Meindl, J.N.; Overley, E.; Robson, K.M. Token Economy: A Systematic Review of Procedural Descriptions. Behav. Modif. 2017, 41, 708-737. [CrossRef] 
15. Dalphonse, A. Token Economy: Examples and Applications in ABA. 2021. Available online: https://masteraba.com/tokeneconomy-2/ (accessed on 25 December 2021).

16. Barreiro-Gomez, J.; Tembine, H. Blockchain Token Economics: A Mean-Field-Type Game Perspective. IEEE Access 2019, 7, 64603-64613. [CrossRef]

17. Drasch, B.J.; Fridgen, G.; Manner-Romberg, T.; Nolting, F.M.; Radszuwill, S. The token's secret: The two-faced financial incentive of the token economy. Electron. Mark. 2020, 30, 557-567. [CrossRef]

18. Narayan, R.; Tidström, A. Tokenizing coopetition in a blockchain for a transition to circular economy. J. Clean. Prod. 2020, 263, 1-9. [CrossRef]

19. Kim, M.; Chung, J. Sustainable Growth and Token Economy Design: The Case of Steemit. Sustainability 2018, 11, 167. [CrossRef]

20. Fisch, C.; Masiak, C.; Vismara, S.; Block, J. Motives and profiles of ICO investors. J. Bus. Res. 2021, 125, 564-576. [CrossRef]

21. Tönnissen, S.; Beinke, J.H.; Teuteberg, F. Understanding token-based ecosystems-A taxonomy of blockchain-based business models of start-ups. Electron. Mark. 2020, 30, 307-323. [CrossRef]

22. Zhao, H.; Cui, W.; Li, S.; Xu, R. Token Economy: A New Form Economy with Decentralized Mutual Trust and Collective Governance. In Proceedings of the IEEE International Symposium on Autonomous Decentralized System, Utrecht, The Netherlands, 8-10 April 2019; pp. 1-7. [CrossRef]

23. FINMA. FINMA Publishes ICO Guidelines; FINMA: Bern, Switzerland, 2018. Available online: https://www.finma.ch/en/news/ 2018/02/20180216-mm-ico-wegleitung (accessed on 25 December 2021).

24. Jung, S.Y.; Kim, T.; Hwang, H.J.; Hong, K. Mechanism Design of Health Care Blockchain System Token Economy: Development Study Based on Simulated Real-World Scenarios. J. Med. Internet Res. 2021, 23, e26802. [CrossRef]

25. Takemiya, M. Sora: A Decentralized Autonomous Economy. In Proceedings of the IEEE International Conference on Blockchain and Cryptocurrency, Seoul, Korea, 14-17 May 2019; pp. 95-98. [CrossRef]

26. Galvagno, M.; Dalli, D. Theory of value co-creation. A systematic literature review. Manag. Serv. Qual. 2014, 24, 643-683. [CrossRef]

27. Krenz, P.; Basmer-Birkenfeld, S.V.; Buxbaum-Conradi, S.; Redlich, T.; Wulfsberg, J. Facing the conflict of transparency and non-disclosure of knowledge within value creation networks. In Proceedings of the 2015 Second International Conference on eDemocracy \& eGovernment (ICEDEG), Quito, Ecuador, 8-10 April 2015; pp. 26-33. [CrossRef]

28. Tyagi, S.; Chambers, T.; Yang, K. Enhanced fuzzy-analytic hierarchy process. Soft Comput. 2018, 22, 4431-4443. [CrossRef]

29. Agarwal, S.; Qian, W. Consumption and Debt Response to Unanticipated Income Shocks: Evidence from a Natural Experiment in Singapore. SSRN Electron. J. 2014, 104, 4205-4230. [CrossRef]

30. Wikepedia. Marginal Propensity to Consume. 2022. Available online: https://en.wikipedia.org/wiki/Marginal_propensity_to_ consume (accessed on 25 December 2021).

31. DeYong, G.D. The price-setting newsvendor: Review and extensions. Int. J. Prod. Res. 2020, 58, 1776-1804. [CrossRef] 